PROCEEDINGS OF THE

AMERICAN MATHEMATICAL SOCIETY

Volume 126, Number 9, September 1998, Pages 2721-2724

S $0002-9939(98) 04636-X$

\title{
REGULARIZATION OF SEMIGROUPS THAT ARE STRONGLY CONTINUOUS FOR $t>0$
}

\author{
P. C. KUNSTMANN
}

(Communicated by Palle E. T. Jorgensen)

\begin{abstract}
Let $E$ be a Banach space and $T:] 0, \infty[\rightarrow L(E)$ a strongly continuous semigroup with $\bigcap_{t>0} \operatorname{Kern} T_{t}=\{0\}$. We show that the generator $A$ of $\left(T_{t}\right)$ generates a regularized semigroup. Our construction of a regularizing operator uses an existence result of J. Esterle.
\end{abstract}

\section{Introduction and Main Result}

Regularized semigroups are an active field of research. They were independently introduced in [1] and [2]. Some other references on this subject are [7], [9] and especially [3]. However, the problem of their relation to semigroups that are strongly continuous for $t>0$ seems not to have been fully cleared, and the quite natural question posed in [6], of whether any semigroup that is strongly continuous for $t>0$ can be regularized, was still unanswered.

It has been known that strongly continuous semigroups of growth order $\alpha>0$ (see [1], [7]) and semigroups of class $\left(C_{(k)}\right)$ (see [8], [7]) can be regularized. In $[6]$, the first result was extended to semigroups whose norms grow not faster than $\exp \left(\omega\left(t^{-1}\right)\right)$ as $t \rightarrow 0$, where $\omega$ is an increasing function satisfying $\int_{1}^{\infty} t^{-2} \log \omega(t) d t$ $<\infty$.

The purpose of this note is to show that no growth assumptions are needed, thus giving a positive answer to the question mentioned above.

Theorem 1.1 (Main Result). Let $\left(T_{t}\right)_{t>0}$ be a semigroup that is strongly continuous for $t>0$ in a Banach space $E$ satisfying $\bigcap_{t>0} \operatorname{Kern} T_{t}=\{0\}$. Then the generator $A$ of $\left(T_{t}\right)$ generates a regularized semigroup.

Recall that in [6] we defined the generator of $\left(T_{t}\right)$ to be the operator

$$
A:=\left\{(x, y) \in E \times E: \forall s>0: \lim _{t \rightarrow 0} \frac{1}{t}\left(T_{t+s} x-T_{s} x\right)=T_{s} y\right\},
$$

which is single-valued, linear and closed and satisfies

$$
A=\left\{(x, y): \forall s>0:\left(T_{s} x, T_{s} y\right) \in A_{0}\right\},
$$

where $A_{0}=\left\{(x, y): \lim _{t \rightarrow 0}\left(T_{t} x-x\right) / t=y\right\}$ is the infinitesimal generator of $\left(T_{t}\right)$ introduced in [5].

The author wants to thank D. Müller for an enlightening hint which finally led to this work.

Received by the editors February 7, 1997.

1991 Mathematics Subject Classification. Primary 47D03, 47D06.

(C)1998 American Mathematical Society 


\section{Proof of the Main Result}

Let $E$ be a Banach space, let $T:] 0, \infty[\rightarrow L(E)$ be a strongly continuous semigroup satisfying $\bigcap_{t>0} \operatorname{Kern} T_{t}=\{0\}$, and let $A$ denote the generator of $\left(T_{t}\right)$. Moreover we assume without loss of generality that the type of $\left(T_{t}\right)$ is negative. Concerning the generator $A$ of $\left(T_{t}\right)$, we remark that

$$
(x, y) \in A \Longleftrightarrow \forall t>s>0: T_{t} x-T_{s} x=\int_{s}^{t} T_{r} y d r .
$$

For the definitions and properties of regularized semigroups we refer to [3]. Recall that a regularized semigroup is a strongly continuous mapping $S:[0, \infty[\rightarrow L(E)$ satisfying $S_{s} S_{t}=S_{t+s} S_{0}$ for all $s, t \geq 0$ and $\operatorname{Kern} S_{0}=\{0\}$, and that its generator $B$ is the closed linear operator defined by

$$
B:=\left\{(x, y): \lim _{t \rightarrow 0}\left(S_{t} x-S_{0} x\right) / t=S_{0} y\right\} .
$$

If the operator $S_{0}$ is to be specified then $\left(S_{t}\right)$ is called an $S_{0}$-regularized semigroup. Recall also that

$$
(x, y) \in B \Longleftrightarrow \forall t>0: S_{t} x-S_{0} x=\int_{0}^{t} S_{r} y d r .
$$

We start with the following lemma.

Lemma 2.1. The generator $A$ of $\left(T_{t}\right)$ generates a regularized semigroup if and only if there is an injective $C \in L(E)$ which commutes with all $T_{t}, t>0$, and satisfies

$$
\operatorname{im} C \subset \Sigma:=\left\{x \in E: \lim _{t \rightarrow 0} T_{t} x=x\right\}
$$

(in which case $A$ generates a $C$-regularized semigroup).

Proof. If $A$ generates a regularized semigroup $\left(S_{t}\right)$, then $C:=S_{0}$ has the desired properties since in this case $T_{t}=S_{0}^{-1} S_{t}$ for all $t>0$ (see e.g. [3]).

On the other hand, if $C$ is as in the assertion, then $S_{0}:=C, S_{t}:=T_{t} C$ for $t>0$, defines a regularized semigroup. Let $B$ denote the generator of $\left(S_{t}\right)$. If $(x, y) \in B$, then we get from (2) that $C\left(T_{t} x-T_{s} x\right)=S_{t} x-S_{s} x=\int_{s}^{t} S_{r} y d r=C \int_{s}^{t} T_{r} y d r$. By the injectivity of $C$ and (1) this implies $(x, y) \in A$. On the other hand, if $(x, y) \in A$, then by applying $C$ to both sides of the right-hand side of (1) and using the definition and the strong continuity of $\left(S_{t}\right)$ we get $(x, y) \in B$ by (2). Hence $A=B$.

We will now construct a suitable regularizing operator $C$ for $\left(T_{t}\right)$. Our construction will use a result of J. Esterle on the existence of functions which generate dense principal ideals in certain convolution algebras.

We first choose a decreasing continuous function $w:] 0, \infty[\rightarrow[1, \infty[$ such that $w(t) \geq\left\|T_{t}\right\|$ for all $t>0$. This is possible since the type of $\left(T_{t}\right)$ is negative.

Let $L^{1}(w)$ denote the Banach space of all measurable complex valued functions $f$ on $] 0, \infty\left[\right.$ such that $\|f\|_{w}:=\int_{0}^{\infty}|f(t)| w(t) d t$ is finite. Since $w \geq 1$ is decreasing, $L^{1}(w)$ is a Banach algebra with respect to convolution.

Lemma 2.2. (a) The mapping $f \mapsto \int_{0}^{\infty} f(t) T_{t} d t$, where the integral is taken in the strong sense, defines a continuous algebra homomorphism $\tilde{T}: L^{1}(w) \rightarrow L(E)$.

(b) For every $f \in L^{1}(w)$ the operator $\tilde{T}(f)$ commutes with all $T_{t}, t>0$, and satisfies $\operatorname{im} \tilde{T}(f) \subset \Sigma$. 
(c) If $f \in L^{1}(w)$ is such that the set of right translates $\left\{\tau_{t} f: t>0\right\}$ is total in $L^{1}(w)$, then $\tilde{T}(f)$ is injective.

Proof. (a) is clear from the choice of $w$ and the semigroup property. For (b) and (c), observe first that $T_{t} \tilde{T}(f)=\tilde{T}\left(\tau_{t} f\right)$ for all $t>0$. Since $w$ is decreasing, the right translation $\left(\tau_{t}\right)$ is by dominated convergence strongly continuous on $L^{1}(w)$. This and (a) prove (b). To prove (c), assume that $\tilde{T}(f) x=0$ and let $x^{*} \in E^{*}$. Then

$$
\int \tau_{t} f(s)\left\langle x^{*}, T_{s} x\right\rangle d s=\left\langle x^{*}, \tilde{T}\left(\tau_{t} f\right) x\right\rangle=\left\langle x^{*}, T_{t} \tilde{T}(f)\right\rangle=0
$$

for every $t>0$. Since $t \mapsto\left\langle x^{*}, T_{t} x\right\rangle$ is a continuous linear functional on $L^{1}(w)$, the assumption on $f$ implies $\left\langle x^{*}, T \cdot x\right\rangle=0$. By Hahn-Banach this means T.x $=0$, and the assumption on $\left(T_{t}\right)$ implies $x=0$.

We give another formulation of the condition in (c).

Remark 2.3. Let $f \in L^{1}(w)$. Then $\left\{\tau_{t} f: t>0\right\}$ is total in $L^{1}(w)$ if and only if $f * L^{1}(w)$ is dense in $L^{1}(w)$.

Proof. If $t>0$ then $\tau_{t} f=\lim _{n \rightarrow \infty} f * \tau_{t} \varphi_{n}$, where $\varphi_{n}:=n \varphi(n \cdot)$ and $\varphi \geq 0$ with $\operatorname{supp} \varphi \subset] 0, \infty\left[\right.$ and $\int \varphi=1$. This clearly holds in $L^{1}$, and also in $L^{1}(w)$ since on the space of functions with support in $[t, \infty[$ both norms are equivalent (recall that $w$ is decreasing and $\geq 1$ ).

On the other hand, let $g$ be continuous with compact support in $] 0, \infty[$. Then $g \in L^{1}(w)$, and the function $\left.h:\right] 0, \infty\left[\rightarrow L^{1}(w), t \mapsto\left(\tau_{t} f\right) g(t)\right.$ is continuous with compact support, hence integrable. Thus we have $f * g=\int_{0}^{\infty} h(t) d t$, which shows that $f * g$ belongs to the closure of the linear span of $\left\{\tau_{t} f: t>0\right\}$. Since right translation is continuous in $L^{1}(w)$, the set of continuous functions with compact support in $] 0, \infty\left[\right.$ is dense in $L^{1}(w)$. The continuity of $*$ in $L^{1}(w)$ implies that we can replace $g$ above by any $g \in L^{1}(w)$.

Now we use the following result ([4], Theorem 6.7) to finish the proof of our theorem.

Theorem 2.4. There is a function $f \in L^{1}(w)$ such that $f * L^{1}(w)$ is dense in $L^{1}(w)$.

Actually, Theorem 6.7 in [4] stated even more, but this version suffices for our purpose. Take $f \in L^{1}(w)$ as in Theorem 2.4; then by Lemma $2.2 C:=\tilde{T}(f)$ satisfies all the conditions in Lemma 2.1, and thus $A$ generates a $C$-regularized semigroup.

Remark 2.5. (a) The assumption $\bigcap \operatorname{Kern} T_{t}=\{0\}$ in our Main Result is also necessary for the existence of an operator $C \in L(E)$ such that $C T_{t}=T_{t} C$ defines a regularized semigroup.

(b) The regularized semigroup we constructed is of the form $\left(\tilde{T}\left(\tau_{t} f\right)\right)$ for a certain $f \in L^{1}(\omega)$. Since $\left(\tau_{t}\right)$ is a $C_{0}$-semigroup in $L^{1}(\omega)$ and $\tilde{T}$ is continuous, we even get a norm-continuous regularized semigroup.

\section{REFERENCES}

[1] G. Da Prato, Semigruppi Regolarizzabili, Ricerche di Mat. 15 (1966), 223-248. MR 37:793

[2] E.B. Davies, M.M.H. PAng, The Cauchy Problem and a Generalization of the Hille-Yosida Theorem, Proc. London Math. Soc. 55 (1987), 181-208. MR 88e:34100

[3] R. deLaubenfels, "Existence Families, Functional Calculi and Evolution Equations," Lect. Notes in Math. 1570, Springer, 1994. MR 96b:47047 
[4] J. Esterle, Elements for a classification of commutative radical Banach algebras, 4-65 in "Radical Banach Algebras and Automatic Continuity", ed. by J.M. Bachar, W.G. Bade, P.C. Curtis Jr., H. G. Dales, M.P. Thomas, Lect. Notes in Math. 975, Springer, 1983. MR 84h:46064

[5] E. Hille, R. S. Phillips, "Functional Analysis and Semigroups," Amer. Math. Soc., Providence, 1957. MR 19:664d

[6] P.C. Kunstmann, A new class of regularizable strongly continuous semigroups of fast growth near the origin, submitted.

[7] I. Miyadera, N. Tanaka, Exponentially bounded $C$-semigroups and generation of semigroups, J. Math. Analysis Appl. 143 (1989), 358-378. MR 90k:47087

[8] S. Oharu, Semigroups of Linear Operators in a Banach Space, Publ. RIMS, Kyoto Univ. 7 (1971/72), 205-260. MR 47:885

[9] N. Tanaka, I. Miyadera, C-Semigroups and the abstract Cauchy problem, J. Math. Analysis Appl. 170 (1992), 196-206. MR 93j:47061

Mathematisches Seminar der Universität Kiel, Ludewig-Meyn-Strasse 4, D-24098 Kiel, Germany

Current address: Mathematisches Institut I der Universität Karlsruhe, Englerstraße 2, Postfach 6980, D-76128 Karlsruhe, Germany

E-mail address: peer.kunstmann@math.uni-karlsruhe.de 